\title{
Charge resolution of the ISS-CREAM SCD measured with a heavy-ion beam
}

\author{
G.H. Hong ${ }^{*+1}$, Y. Amare ${ }^{2}$, T. Anderson ${ }^{3}$, D. Angelaszek ${ }^{2,4}$, N. Anthony ${ }^{2}$, K. \\ Cheryian $^{2}$, G.H. Choi ${ }^{1}$, M. Copley ${ }^{2}$, S. Coutu ${ }^{3}$, L. Derome ${ }^{5}$, L. Eraud ${ }^{5}$, L. Hagenau \\ ${ }^{2}$, J.H. Han ${ }^{2}$, H.G. Huh ${ }^{2}$, Y.S. Hwang ${ }^{6}$, H.J. Hyun ${ }^{\ddagger 6}$, S. Im ${ }^{3}$, H.B. Jeon ${ }^{6}$, J.A. Jeon ${ }^{1}$, \\ S. Jeong ${ }^{1}$, S.C. Kang ${ }^{6}$, H.J. Kim ${ }^{6}$, K.C. Kim ${ }^{2}$, M.H. Kim ${ }^{2}$, H.Y. Lee ${ }^{1}$, J. Lee ${ }^{1}$, M.H. \\ Lee $^{\S 2}$, J. Liang ${ }^{2}$, J.T. Link ${ }^{\mathbb{7}}$, L. Lu ${ }^{2}$, L. Lutz ${ }^{2}$, A. Menchaca-Rocha ${ }^{8}$, T. Mernik ${ }^{2}$, \\ J.W. Mitchell ${ }^{7}$, S.I. Mognet ${ }^{3}$, S. Morton ${ }^{2}$, M. Nester ${ }^{2}$, S. Nutter ${ }^{9}$, O. Ofoha ${ }^{2}$, H. \\ Park $^{6}$, I.H. Park ${ }^{1}$, J.M. Park ${ }^{6}$, N. Picot-Clemente ${ }^{2}$, R. Quinn ${ }^{2}$, E.S. Seo ${ }^{2,4}$, J.R. \\ Smith $^{2}$, P. Walpole ${ }^{2}$, R.P. Weinmann ${ }^{2}$, J. Wu ${ }^{2}$, Y.S. Yoon ${ }^{\mid 2,4}$ \\ (1) Sungkyunkwan University, Natural Sciences Campus, Dept. of Physics, Suwon 16419, \\ Republic of Korea \\ $\left(^{2}\right)$ Inst. for Phys. Sci. and Tech., University of Maryland, College Park, MD 20742, USA \\ $\left.{ }^{3}\right)$ Dept. of Physics, Penn State University, University Park, PA 16802, USA \\ $\left({ }^{4}\right)$ Dept. of Physics, University of Maryland, College Park, MD 20742, USA \\ $\left.{ }^{5}\right)$ Laboratoire de Physique Subatomique et de Cosmologie, UJF - CNRS/IN2P3 - INP, 53 rue \\ des Martyrs, 38026 Grenoble Cedex, France \\ ${ }^{6}{ }^{\prime}$ Dept. of Physics, Kyungpook National University, Deagu 702-701, Republic of Korea \\ ( ${ }^{7}$ ) Astrophysics Space Division, NASA Goddard Space Flight Center, Greenbelt, MD 20771, USA \\ $\left.{ }^{8}\right)$ Instituto de Fisica, Universidad Nacional Autonoma de Mexico, Circuito de la Investigación \\ s/n,Ciudad Universitaria, CP 04510 México Distrito Federal, Mexico \\ $\left({ }^{9}\right)$ Dept. of Physics and Geology, Northern Kentucky University, Highland Heights, KY 41076, \\ USA \\ E-mail: hgh987321@skku.edu
}


The Cosmic Ray Energetics And Mass experiment for the International Space Station (ISSCREAM) is scheduled to be launched and installed on the ISS in August 2017, and will carry out a measurement of the energy and composition of energetic cosmic rays in space. The Silicon Charge Detector (SCD) will identify the charge of through-going cosmic rays. It consists of four layers, each with 2688 silicon pixels and associated electronics. The ISS-CREAM payload was delivered to the launch site, Kennedy Space Center, in August 2015 after the successful completion of integration and space environment tests. A heavy-ion beam, required to verify the capability of precision charge measurement of the SCD, became available at the European Organization for Nuclear Research (CERN) in November 2016. A prototype instrument using the same types of silicon pixel sensors and electronics installed in the SCD was placed in a heavy-ion beam composed of secondary ions ranging from helium to zinc. We present the charge resolution for each ion as a function of the number of layers used for charge measurement so that the improvement in charge resolution is clearly demonstrated as the number of layers for charge measurement increases.

35th International Cosmic Ray Conference - ICRC2017

10-20 July, 2017

Bexco, Busan, Korea

\footnotetext{
* Speaker.

${ }^{\dagger}$ for the ISS-CREAM Collaboration(http://cosmicray.umd.edu/iss-cream/collaboration)

${ }^{\ddagger}$ Currently at Pohang Accelerator Laboratory, Jigokro-127-beongil, Nam-gu, Pohang 37673, Republic of Korea

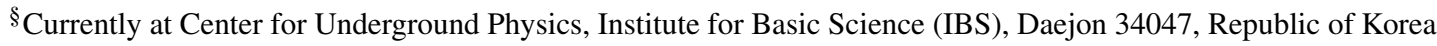

IAlso at CRESST/USRA, Columbia MD 21044, USA

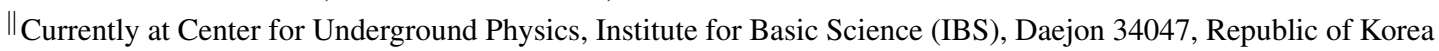




\section{Introduction}

The origin of cosmic rays, and their acceleration and propagation mechanisms have remained unknown since their discovery a century ago. Various suborbital and space experiments have been carrying out their missions to study the cosmic rays in the energy range of up to $10^{15} \mathrm{eV}$ in the effort to answer the century old question. The Cosmic Ray Energetics And Mass (CREAM), an Antarctica balloon-borne experiment, is one of the suborbital experiments. It has successfully completed seven flights between the years 2004 and 2017, and it collected data for a total of 191day exposure during these flights. It discovered a discrepant hardening in the energy spectra of cosmic rays [1]. However, high-statistics measurements of energetic cosmic rays are required for further investigation of this unexpected hardening. The International Space Station (ISS) is an ideal platform to further investigate the details of the energy spectra as well as explore fundamental issues like the acceleration mechanism and the origin of the energetic cosmic rays, because of the high duty cycle of experiments on the ISS platform. Thus, the space-borne ISS-CREAM experiment to be deployed to the ISS is an obvious next step towards further investigation and exploration of the outstanding issues with high statistics. The ISS-CREAM experiment adapts the detector concept and technology proven with the balloon-borne CREAM experiment. Two major instruments of the ISS-CREAM payload are the calorimeter for energy measurement and the Silicon Charge Detector (SCD) for elemental identification via charge measurement of cosmic rays. The SCD has four layers of silicon pixel sensors and associated electronics, and it is expected to measure the charge of cosmic rays up to $Z=26$ with a charge resolution of 0.2 charge units or better. It required heavy ions to confirm the range of charge measurement and its resolution. A beam of heavy ions became available in November 2016 at CERN. Primary lead ions hit a target to produce secondary heavy ions from the fragmentation process upstream of the beam test location. Heavy ions with $A / Z=2$ and a momentum of $60 \mathrm{GeV} / \mathrm{c}$ per nucleon, then, were focused into a beam of $1 \mathrm{~cm}$ size at the beam test location. The heavy-ion beam was composed of ions ranging from helium to zinc. We built a prototype of the SCD using the same silicon pixel sensors and electronics used in the ISS-CREAM SCD to carry out the beam test. We present the charge resolution of heavy ions as a function of the number of layers used for charge measurement.

\section{Experimental setup for the heavy-ion beam test}

The heavy-ion beam test was carried out at the $\mathrm{H} 4$ and $\mathrm{H} 8$ beam lines where the primary lead beam was extracted from the Super Proton Synchrotron (SPS) accelerator at CERN. The experimental setup for the heavy-ion beam test had two parts: a trigger detector and the prototype SCD instrument. The left-hand side of Fig. 1 shows a sketch of the setup, with the right-hand side a photo of the setup installed in the beam test site. The experimental setup was installed on a movable stage, as shown in the right-hand side of Fig. 1, which was controlled remotely from outside of the beam line. The trigger detector was placed in front of a movable stage. The position of the movable stage was adjusted until the sensors inside the experimental setup were aligned with the heavy-ion beam. 

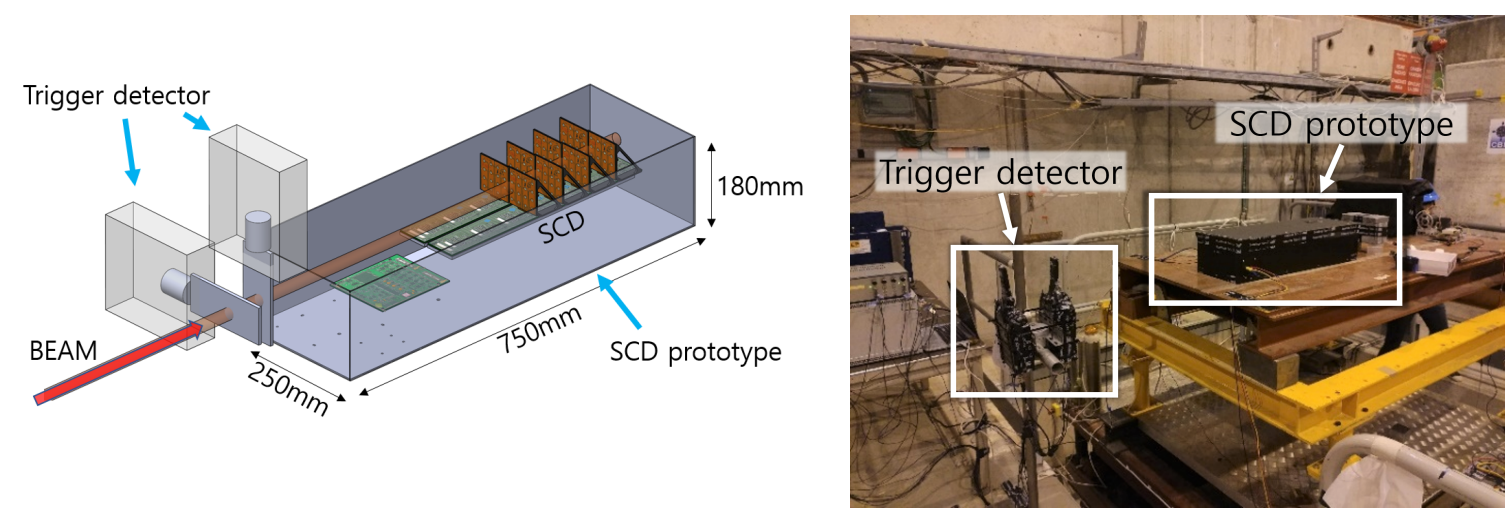

Figure 1: Sketch (left) and photo (right) of the setup for the heavy-ion beam test.

\section{Instrument}

The trigger detector consisted of two plastic scintillators with 1-inch photomultiplier tubes attached, and two discriminators and an AND gate of the Nuclear Instrumentation Modules (NIM) standard. The two plastic scintillators, each of area $15 \times 5 \mathrm{~cm}^{2}$, were placed $20 \mathrm{~cm}$ apart upstream of the SCD prototype to trigger on heavy ions incident on them. The scintillators responded to heavy ions to produce scintillation light which was converted to photoelectrons at photocathodes of the photomultiplier tubes. Then the photoelectrons were multiplied in the photomultiplier tubes to generate fast current signals. These signals were fed to two separate NIM discriminators which generated logic pulses if the input signals exceeded preset the preset thresholds. The two logic pulses were then fed to a NIM AND gate to generate a logic pulse when the signals from two scintillators were coincident with each other. The trigger logic pulse was finally fed to the SCD prototype to trigger heavy-ion events. The SCD prototype instrument is composed of silicon pixel sensors and readout electronics which were of the same types as the ones installed in the ISSCREAM SCD. To block ambient light and electromagnetic noise, all silicon sensors and readout electronics were placed inside an aluminum light-tight box as shown in Fig. 2. The SCD prototype instrument has the same 4-layer configuration as the ISS-CREAM SCD. Each layer of the prototype has two silicon pixel sensors side by side as shown in Fig. 2. A silicon sensor has 16 pixels in a 4 $\times 4$ array, and each pixel has an area of $15.5 \times 13.7 \mathrm{~mm}^{2}$ and a thickness of $525 \mu \mathrm{m}$. A total of 8 silicon sensors were placed in the prototype. To have the 4-layer configuration while minimizing the volume of the prototype and the number of readout channels, the silicon sensors were placed straight up, orthogonal to the analog electronics boards. The silicon sensors were supported at their edges by aluminum brackets to stand straight up. The readout electronics of the SCD prototype instrument consists of three parts: analog, digital, and data acquisition electronics. The analog electronics is based on the CR1.4 ASIC chips [2]. Each CR1.4 chip has 16 channels, and each channel has a preamplifier followed by a shaper. The preamplifier converts the current signal from a silicon pixel to a voltage signal, and the shaper transforms the voltage signal to a signal with shape appropriate for sample and hold. When each chip receives a trigger signal, it holds the voltages of the shaped signals at the time of trigger for all 16 channels, and multiplexes the voltages of the 16 channels into an output signal. In one Printed Circuit Board (PCB) of analog electronics, there are 

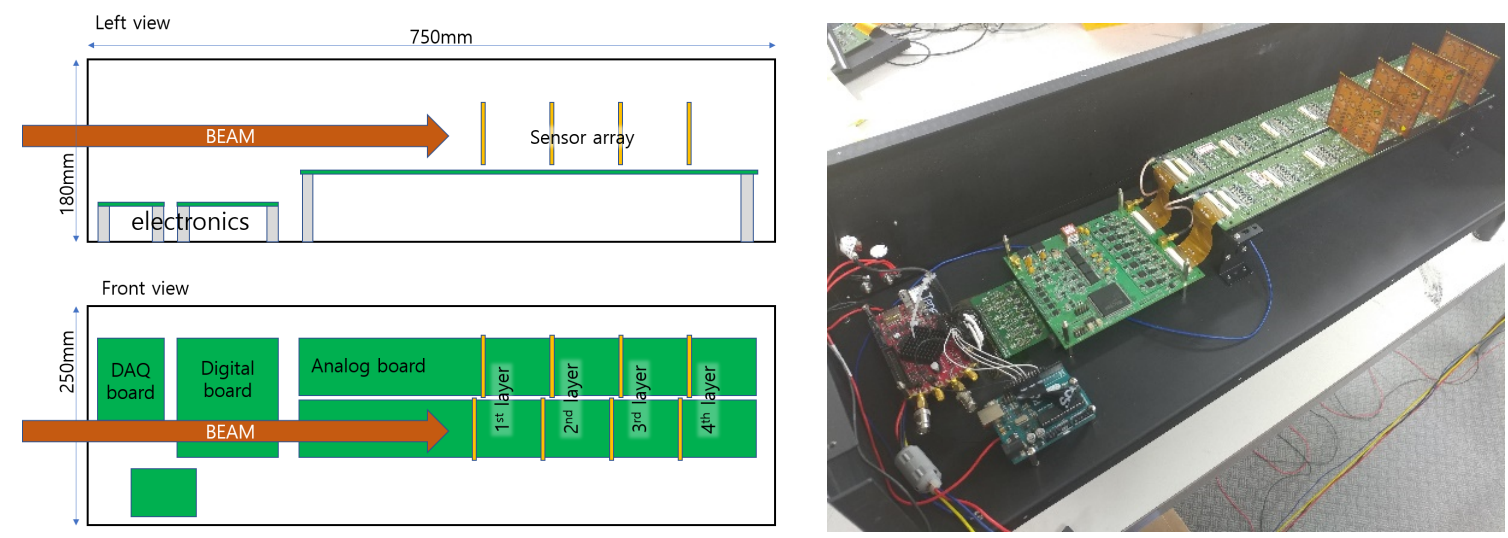

Figure 2: Sketch (left) and photo (right) of the SCD prototype instrument for the heavy-ion beam test.

7 CR1.4 chips mounted. The prototype instrument has a total of 2 analog electronics boards, each only with 4 CR1.4 chips connected to silicon sensors. Multiplexed analog voltage outputs from the analog electronics are converted to digital values in digital electronics. The PCB of the digital electronics is equipped with an Analog to Digital Converter (ADC) chip, control interfacing parts, various power regulators and converters. The digital electronics board receives DC voltages of +6 and $-6 \mathrm{~V}$ from external power supplies, and regulates and distributes them to two analog electronics boards. It also receives a $12 \mathrm{~V}$ voltage from an external power supply, and the voltage is input to a DC-DC converter which produces a $140 \mathrm{~V}$ voltage for biasing silicon sensors. The prototype instrument adopted a simple data acquisition method instead of using the complex and sophisticate data acquisition system of ISS-CREAM. The electronics board for the simple data acquisition is equipped with a Field Programmable Gate Array (FPGA) chip and various interfacing chips for control and data transfer. The FPGA chip was programmed to start the full chain of data readout upon receiving the trigger signal and transfer digitized data to the external PC via a Serial Peripheral Interface (SPI) bus. Since the dead time caused by the data transfer is about $25 \mathrm{~ms}$, the maximum trigger rate of the entire system was about $40 \mathrm{~Hz}$.

\section{Data analysis}

The Bethe-Bloch formula relates the mean energy loss $\frac{d E}{d x}$ of ions in a material to the charge $\mathrm{Z}$ of the ions as follows: $\left\langle\frac{d E}{d x}>=I Z^{2}\right.$ or $Z=\sqrt{<\frac{d E}{d x}>/ I}$. We converted the detector response (i.e., the energy loss $\frac{d E}{d x}$ ) to the charge according to the Bethe-Bloch formula using the value of I evaluated for Fe ions. To study the dependence of the charge resolution of the SCD on the number of layers used for charge measurement, we compared the single-layer, double-layer, triple-layer and quadruple-layer charge measurements. Shown in Fig. 3 is the 2-dimensional scatter plot of heavy-ion charges measured with the first layer $\left(Z_{1}\right.$ on $y$-axis) and second layer $\left(Z_{2}\right.$ on $x$-axis). The single-layer charge distribution was obtained by projecting the 2-dimensional plot onto the $y$-axis. The double-layer charge distribution was obtained by selecting the central band of the 2dimensional plot and taking the average of $\mathrm{Z}_{1}$ and $\mathrm{Z}_{2}$. The triple-layer and quadruple-layer charge measurements were obtained by selecting such central bands of 2-dimensional plots for appropriate 


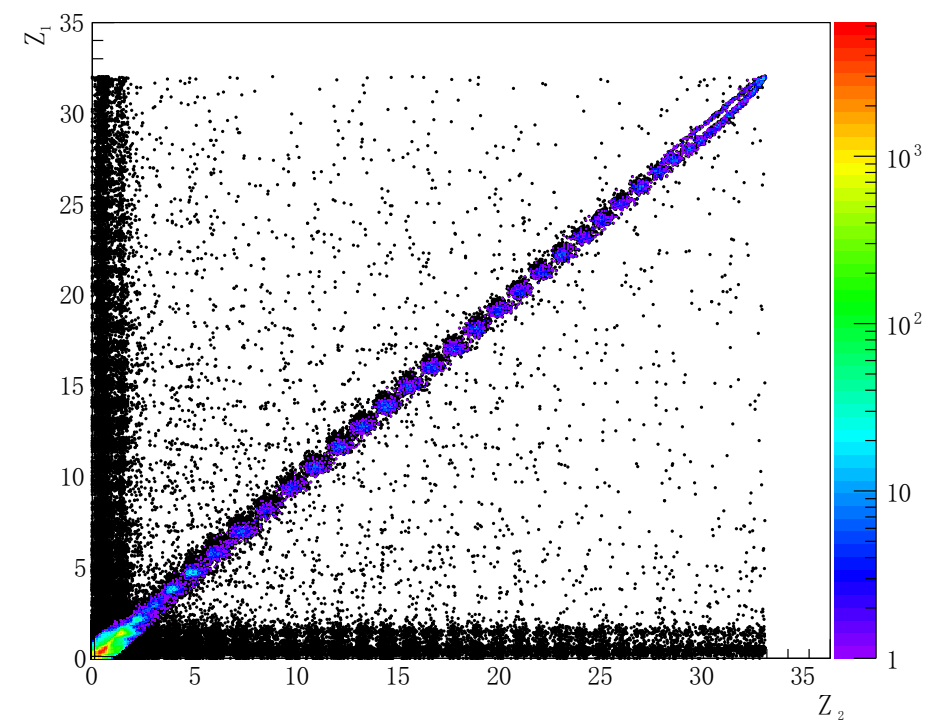

Figure 3: Two-dimensional scatter plot of heavy ion charges measured with the first layer (y-axis) and second layer (x-axis).

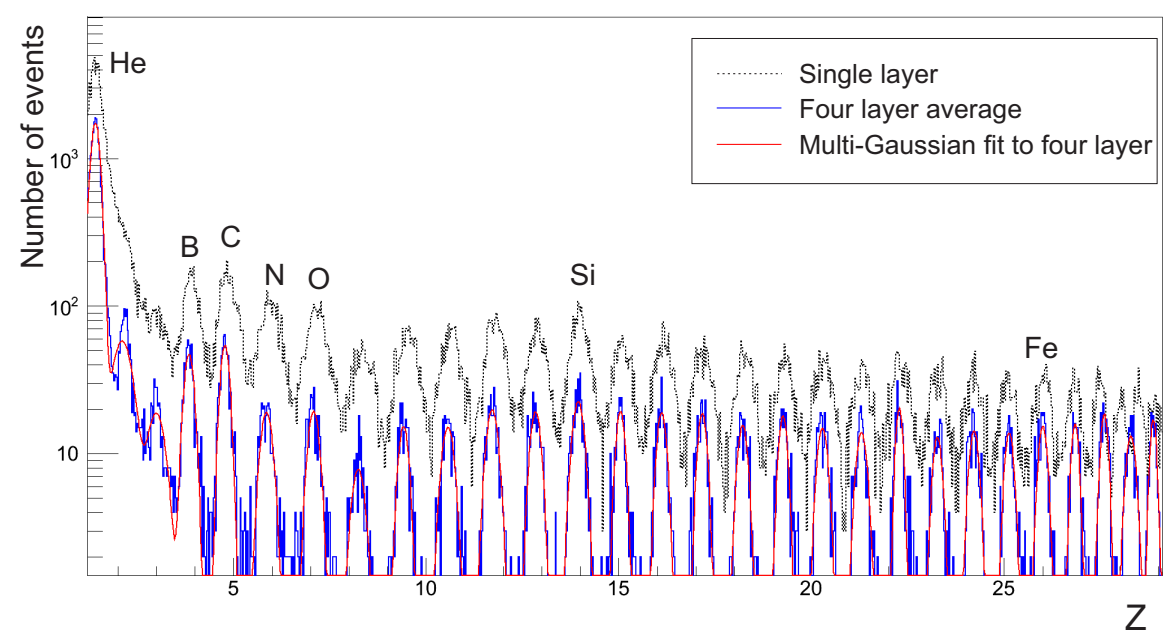

Figure 4: Charge distribution of heavy ions: the dotted histogram is the distribution measured with a single layer while the blue histogram is the distribution measured with all four layers. The red curve is the multiGaussian fit to the charge distribution measured with all four layers.

combinations of charge measurements and taking the average of the involved charge measurements. The dotted histogram in Fig. 4 is the distribution of heavy-ion charges measured with a single layer while the blue histogram is the distribution measured with all four layers. The red curve in Fig. 4 is the multi Gaussian fit to the distribution measured with four layers. 


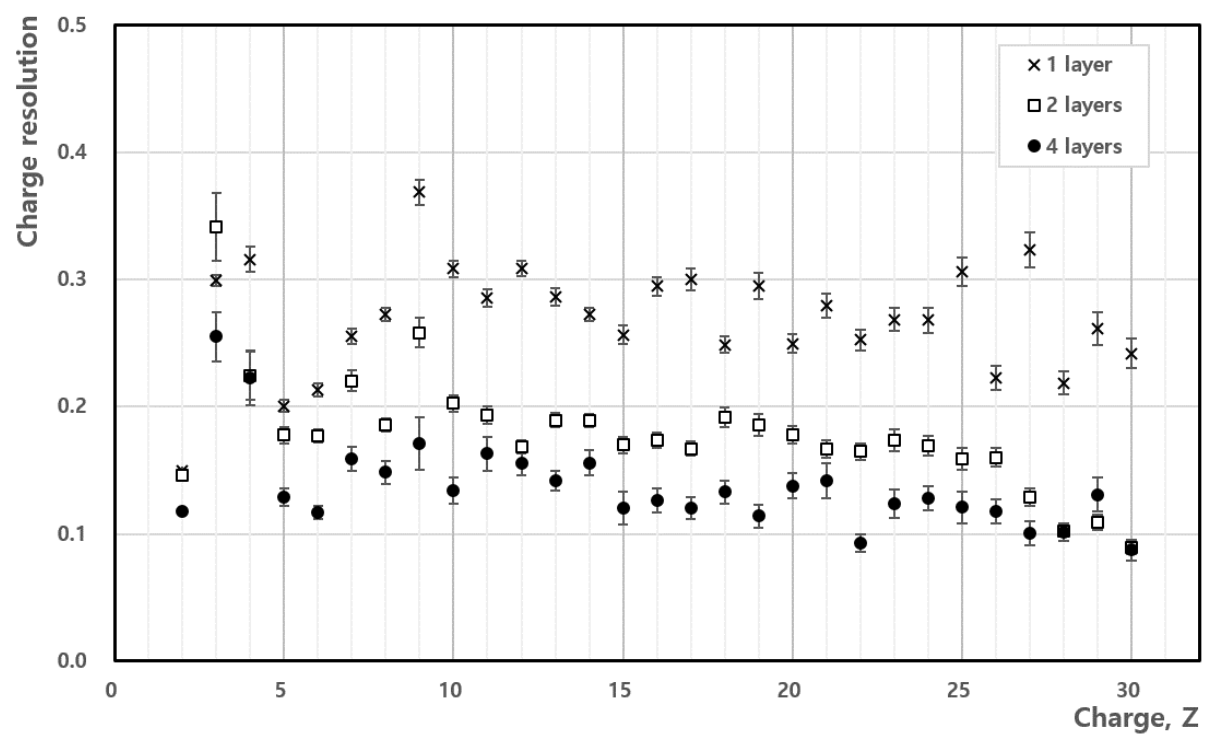

Figure 5: Charge resolution of the ions from He through $\mathrm{Zn}$ measured with different numbers of layers.

\begin{tabular}{c|c|c|c}
\hline \multirow{2}{*}{} & \multicolumn{3}{|c}{ Charge resolution (in charge unit) } \\
\cline { 2 - 4 } & 1 layer & 2 layer & 4 layer \\
\hline \hline $\mathrm{C}$ & 0.213 & 0.177 & 0.117 \\
\hline $\mathrm{N}$ & 0.255 & 0.220 & 0.159 \\
\hline $\mathrm{O}$ & 0.272 & 0.186 & 0.148 \\
\hline $\mathrm{Si}$ & 0.273 & 0.189 & 0.156 \\
\hline $\mathrm{Fe}$ & 0.222 & 0.160 & 0.118 \\
\hline Average & 0.270 & 0.178 & 0.137 \\
\hline
\end{tabular}

Table 1: Charge resolutions of $\mathrm{C}, \mathrm{N}, \mathrm{O}, \mathrm{Si}$ and $\mathrm{Fe}$, and average charge resolution measured with different numbers of layers.

\section{Result}

Shown in Fig. 5 are the charge resolutions (in charge units) of the ions from He through $\mathrm{Zn}$ measured with different numbers of layers. The charge resolutions of $\mathrm{C}, \mathrm{N}, \mathrm{O}, \mathrm{Si}$ and Fe measured with different numbers of layers are listed in Table 1. Also the average charge resolutions over the ions from He through $\mathrm{Zn}$ measured with different numbers of layers are also listed in Table 1. The charge resolution improves as the number of layers increases. It is statistically expected that the charge resolution measured with all four layers is half of the resolution measured with a single layer. The single-layer charge resolution averaged over all ions is 0.270 in charge units. The quadruple-layer charge resolution over all ions is 0.137 in charge units, which is very close to the statistically expected resolution of 0.135 in charge units. 


\section{Conclusion}

The ISS-CREAM SCD was designed to measure the charge of cosmic rays from $Z=1$ to $Z=30$. To confirm the performance of the SCD charge measurement, a heavy-ion beam test was carried out at the European Organization for Nuclear Research (CERN) in November 2016. It was verified that the charge resolution improves as the number of layers for measurement increases, and the improvement is in good agreement with the statistical expectation.

\section{Acknowledgments}

The authors thank the NASA GSFC WFF for project management and engineering support, and the NASA JSC ISS Program Office for the launch support and the ISS accommodation. This work was supported in the U.S. by NASA grants NNX11AC52G, NNX08AC15G, NNX08AC16G and their predecessor grants, as well as by directed RTOP funds to NASA GSFC WFF. It is supported in Korea by the Creative Research Initiatives of MEST/NRF and by National Research Foundation Grants NRF-2014R1A2A2A01002734, NRF-2014R1A1A2006456, NRF-2015R1A2A1A13001843. It is supported in France by IN2P3/CNRS and CNES and in Mexico by DGAPA-UNAM and CONACYT. The authors also thank H.S. Choi, Korea Institute of Industrial Technology, for contributions to the SCD thermal vacuum tests, M. Geske, Penn State, for contributions to the BSD, and M. A. Coplan, University of Maryland, contributions to CAL electronics vacuum tests.

\section{References}

[1] H. S. Ahn et al., Discrepant hardening observed in cosmic-ray elemental spectra, Astrophys. J 714 (2010), L89-L93.

[2] J. H. Adams et al., The CR-1 chip: custom VLSI circuitry for cosmic rays, Proceedings of the 26th International Cosmic Ray Conference, Salt Lake City, Vol. 5 (1999), 69-71. 